\title{
Hybrid ventilation of a room: a theoretical model for the combined effects of mechanically-imposed and buoyancy-induced driving pressures
}

\author{
Owen Connick ${ }^{1}$ and Gary R. Hunt ${ }^{* 2}$ \\ ${ }^{1}$ Breathing Buildings, 15 Sturton Street, Cambridge, CB1 2SN, UK. \\ ${ }^{2}$ Department of Engineering, University of Cambridge, Trumpington \\ Street, Cambridge CB2 1PZ, UK
}

November 5, 2019

\begin{abstract}
A supply fan and an extract fan, at identical settings in identical rooms, do not necessarily have the same effect on the ventilation. For a heated room in which the floor-level vents are larger than the ceilinglevel vents, we show that an extract fan provides better ventilation than a supply fan. If the ceiling-level vents are larger, we show that a supply fan is more effective; this for the same, constant fan airflow rate. We investigate the hybrid ventilation of a room in which the, otherwise buoyancy-driven, ventilation is augmented by a forced volume flux, provided by a supply or extract fan. In hybrid ventilation, naturallyoccurring and mechanically-imposed pressure differences combine to determine the resulting ventilation airflow. Herein, a mathematical model is developed which enables prediction of: the inflow and outflow volume fluxes; the vertical position of the neutral pressure level; and the steady, uniform temperature, within a single, isolated room in hybrid ventilation. The physical problem is reduced to a mathematical model with two controlling parameters; namely, the effective vent area ratio, $R^{*}$, and a ratio of forced and natural volume fluxes, $Q_{F} / Q_{N}$. We demonstrate that the volume flux through an open vent can be controlled remotely, by mechanically imposing the volume flux through an entirely separate vent.
\end{abstract}

\section{Introduction}

Building on the existing understanding of purely natural ventilation, herein we investigate the effect of a mechanically-imposed supply or

\footnotetext{
*Email address for correspondence: gary.hunt@eng.cam.ac.uk
} 
extract fan volume flux, $Q_{F}$, on the otherwise buoyancy-driven ventilation of a room. The resulting ventilation airflow, which is driven by the combined effects of naturally-occurring and mechanically-imposed pressure differences, is referred to as hybrid ventilation.

Since the 1990s numerous studies have been conducted in Europe and Asia focussing on the common goal of developing hybrid ventilation systems that reduce energy expenditure in buildings by reducing our reliance on mechanical ventilation (Kwon et al. 2013). Ji et al. (2009) succinctly capture the difficulties of meeting this goal, highlighting that two of the challenges associated with hybrid ventilation design are determining the performance of the passive system and maximising the period of operation of the passive system. In addition to reducing energy consumption relative to a purely mechanical system, a second attraction of a hybrid approach regards the potential enhancement in airflow control over purely passive systems.

The International Energy Agencys (IEA) Annex 35, HybVent project, entitled Hybrid Ventilation in New and Retrofitted Office Buildings (IEA 1997) provided a catalyst for rapid developments in our understanding of hybrid ventilation. Launched in 1997, the early works under the four year framework were expansive and paved the way for the now considerable body of work on this subject. Whilst we make no attempt here to provide a comprehensive review, detailed literature reviews of methods for analysing hybrid and natural ventilation are given by Li et al. (1999) and Li and Heiselberg (2003); the evaluation of hybrid control strategies are discussed by Delsante and Aggerholm (2002); and the categorising of hybrid ventilation flows by Heiselberg (2002).

These works have been complemented by elegant theoretical modelling studies, including by Li (2002) who describes a model for ventilation of a single room with open vents and a mechanical fan, by computational fluid dynamic (CFD) studies focussing on specific buildings and different climates (e.g. Lomas et al. 2007, Ji et al. 2009, Zhai et al. 2011) and by full-scale measurements on hybrid ventilation performance, e.g. in apartment buildings (Niachou et al. 2008) and atria (Hussain and Oostuizen 2012). In addition to CFD, other simulation tools have been deployed to analyse the performance of hybrid systems as detailed, for example, by Jeong and Haghighat (2002), El Mankibi et al. (2006) and Wang and Chen (2013).

Other studies have brought additional physics into play so as to ultimately widen the practical deployment of hybrid ventilation and further increase energy savings. Examples include investigations of how hybrid ventilation strategies can be integrated with heat storage systems (Kwon et al. 2013), with thermal mass (Wang and Chen 2013) and with the effects of natural wind (Wang et al. 2014). Beyond building ventilation, strategies combining natural and mechanical ventilation are also proving instrumental in the control and removal of smoke resulting from fires in subway stations and tunnels (Gao et al. 2012, Tanaka et al. 2015).

Surveying the available literature reveals that there is considerable 
scope to build upon the published works. There remain a number of fundamental questions regarding the interaction between the naturallydriven and mechanically-driven components of the flow, which motivate the current theoretical work.

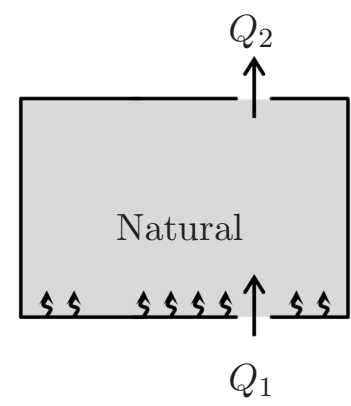

(a)

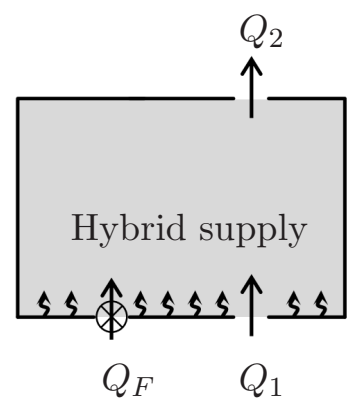

(b)

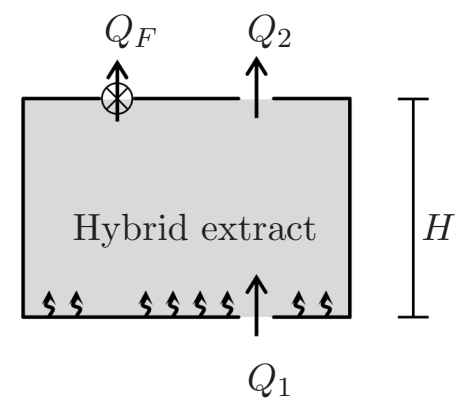

(c)

Figure 1: Schematics of: (a) purely natural ventilation; (b) hybrid supply ventilation; and (c) hybrid extract ventilation. Small arrows near floor level represent a distributed heat input. Straight arrows indicate the direction of flow through ventilation openings, in forward flow. Crossed circles ( () represent a vent at which the volume flux is mechanically fixed.

Our study focusses on a single, isolated room of height $H$, as represented schematically in figure 1 . The room receives a constant supply of heat from a distributed, floor-level heat source, represented by the buoyancy flux, $B$, and has open vents of area $a_{1}$ and $a_{2}$ in the floor and ceiling, respectively. We assume a highly insulated room, so that heat transfer to and from the walls may be neglected, and the only way heat may escape is in the warm air outflow.

When the buoyancy flux into the room (from the heat source) is exactly matched by the buoyancy flux out of the room (carried by the outflow), a steady state will result. This can be expressed as

$$
B=Q_{t o t} g^{\prime},
$$

where $Q_{t o t}$ is the total outflow volume flux and $g^{\prime}$ is the local buoyancy (or reduced gravity) of the outflowing air. This 'steady state' is the condition that is typically designed for and thus is of primary interest, and the focus herein.

The reduced gravity, $g^{\prime}$, is a commonly used measure of the relative buoyancy of warm indoor air compared with the cooler exterior environment. For this reason, $g^{\prime}$ is referred to as the local buoyancy, defined as

$$
g^{\prime}=g \frac{T-T_{0}}{T_{0}},
$$

where $T$ and $T_{0}$ are the absolute temperature of warm inside and cooler outside air, respectively, and $g$ is the acceleration due to gravity. Our 
focus will be on steady hybrid flows and an environment of constant temperature. As such, we make the assumption that the timescale for the development of the flow is short relative to the timescale associated with external temperature changes. The buoyancy flux $B$ can be thought of as a rate of supply of heat and can be related to the power, $P$, of the heat source as follows

$$
B=P \frac{g}{\rho_{0} T_{0} C p}=Q g^{\prime}
$$

where $\rho_{0}$ and $C p$ are the density and specific heat capacity, respectively, of the air being heated (Batchelor, 1954). In (3), $Q$ denotes the volume flow rate of air with buoyancy $g^{\prime}$. Gladstone and Woods (2001) showed that an areally distributed heat source generates high-Rayleighnumber, turbulent convection, resulting in approximately uniform heating of air within a room. This informs our decision to model the space as being at uniform temperature. Based on this assumption, $g^{\prime}$ represents the buoyancy of the air throughout the room.

Mechanically imposing a forced volume flux alters the naturallyoccurring pressure distribution, effecting the airflow through low- and high-level vents differently. Two design cases are considered: hybrid supply ventilation and hybrid extract ventilation, as illustrated in figure $1 \mathrm{~b}$ and figure $1 \mathrm{c}$, respectively. A supply fan increases the air pressure inside a room, whilst an extract fan decreases the air pressure. This is an important distinction. Before investigating hybrid ventilation, it is useful to review purely natural ventilation ( $\$ 2)$. Hybrid supply ventilation and hybrid extract ventilation will be addressed separately in $\S 3$ and $\S 4$, respectively. Whilst in some practical situations there could potentially be a short-circuiting of the flow, as air ejected from the room via one vent enters through another, a working assumption that we make in our theoretical developments is that the locations of mechanical extract and supply, and of the upper and lower vents (figure 1), are such that there is no short circuiting.

To aid those developments, it is convenient here to introduce the ratio of effective vent areas,

$$
R^{*}=\frac{a_{1} c_{1}}{a_{2} c_{2}},
$$

where $a_{1}$ and $a_{2}$ are the areas of the floor-level and ceiling-level vents, respectively, and $c_{1}$ and $c_{2}$ are their respective loss coefficients. This ratio compares the relative sizes of the floor-level and ceiling-level vents. Values of $R^{*}<1$ indicate relatively smaller vents at floor level than at ceiling level. Conversely, values of $R^{*}>1$ indicate relatively larger vents at floor level.

\section{Purely natural ventilation}

In the absence of wind, natural ventilation airflows are driven solely by the effects of buoyancy. Warm air accumulates within the room and, due to its buoyancy, generates a difference in static pressure between air 
inside the room and air outside at the same height. The airflow through any ventilation opening is driven by the static pressure difference across that opening.

Figure $2 \mathrm{a}$ shows a schematic of a uniform temperature, naturally ventilated room. In figure $2 \mathrm{~b}$, the static air pressure both inside, $p_{i}(z)$, and outside, $p_{o}(z)$, the room is illustrated schematically. Figure $2 \mathrm{c}$ shows the variation of the pressure difference, $\Delta p(z)$, over the room height, where $\Delta p(z)=p_{i}(z)-p_{o}(z)$.

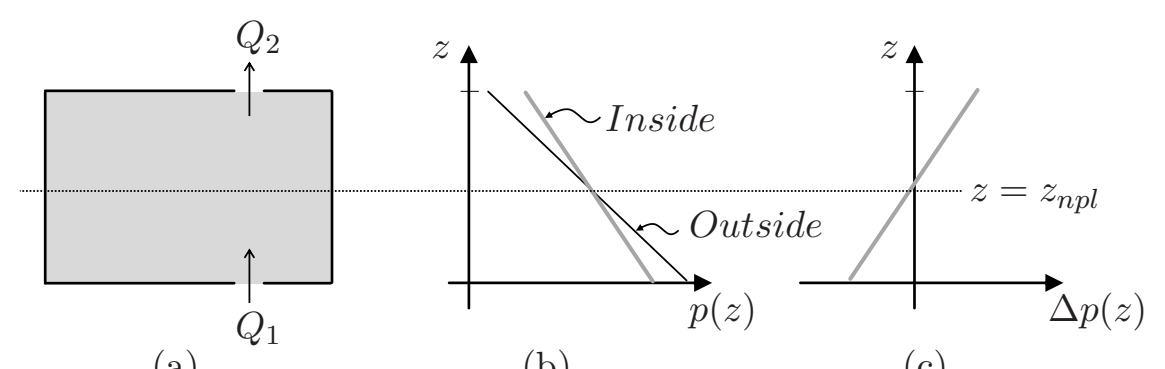

(a)

(b)

(c)

Figure 2: Schematics of: (a) a naturally ventilated room with floor-level, inflow volume flux, $Q_{1}$, ceiling-level, outflow volume flux, $Q_{2}$; (b) the vertical variation in static pressure, $p(z)$, inside and outside the room; and (c) the corresponding vertical variation of pressure difference, $\Delta p(z)$. The horizontal dotted line denotes the vertical position of the neutral pressure level, $z=z_{n p l}$.

By tracing changes in static pressure within a uniformly warm, naturally ventilated room, the balance of pressures may be written as

$$
|\Delta p|_{z=0}+|\Delta p|_{z=H}=\rho_{0} g^{\prime} H,
$$

where $g^{\prime}$ is the uniform local buoyancy of air within the room and $\rho_{0}$ is the constant density of outside air. Considering the density of outside air to be constant neglects the effects of temperature changes over the period of interest - this is considered to be a reasonable approximation. The pressure difference terms, $\Delta p_{z=0}$ and $\Delta p_{z=H}$ are the static pressure differences across the floor-level and ceiling-level vents, respectively. Applying Bernoulli's theorem along streamlines which pass through the floor-level and ceiling-level vents, the pressure difference terms in (5) can be replaced by the volume fluxes, $Q_{1}$ and $Q_{2}$, through those vents. Doing so gives

$$
\frac{Q_{1}^{2}}{2 a_{1}^{2} c_{1}^{2}}+\frac{Q_{2}^{2}}{2 a_{2}^{2} c_{2}^{2}}=g^{\prime} H .
$$

In purely natural ventilation $Q_{1}=Q_{2}=Q_{B}$, where $Q_{B}$ is the buoyancydriven volume flux, and (6) can be reduced to

$$
\frac{Q_{B}^{2}}{A^{* 2}}=g^{\prime} H,
$$


where $A^{*}$ is an effective vent area, given by

$$
\frac{1}{A^{* 2}}=\frac{1}{2 a_{1}^{2} c_{1}^{2}}+\frac{1}{2 a_{2}^{2} c_{2}^{2}} .
$$

The loss coefficients ${ }^{1} c_{1}$ and $c_{2}$, associated with the floor-level and ceiling-level vents, respectively are commonly taken as constants, with value $c_{1}=c_{2}=0.6$ (Etheridge and Sandberg 1996, Linden 1999). This is despite the work of Hunt and Holford (2000) who showed that these coefficients depend upon the area of, the density contrast across, and the volume flux through, the vent in question. In the current work we do not explicitly specify values of $c_{1}$ and $c_{2}$, thereby avoiding the complexities of this topic.

In a uniformly warm room, the local buoyancy of the outflow, $g^{\prime}$ in (1), is the uniform local buoyancy of air within the room. Substituting (1) into (7) allows us to express the steady natural ventilation volume flux as

$$
Q_{N}=\left(A^{* 2} B H\right)^{1 / 3},
$$

the subscript ' $N$ ' reading 'Natural'.

The natural ventilation volume flux, $Q_{N}$, and the buoyancy-driven volume flux, $Q_{B}$, are intentionally defined separately in order to emphasise that, whilst the two are equivalent in purely natural ventilation, in hybrid ventilation they will not be. Further substituting (1) into (9), the uniform local buoyancy in steady natural ventilation can be expressed as

$$
g_{N}^{\prime}=\left(\frac{B^{2}}{A^{* 2} H}\right)^{1 / 3} .
$$

The natural ventilation volume flux, $Q_{N}$, and the resulting local buoyancy, $g_{N}^{\prime}$, depend only on the input buoyancy flux, $B$, the height of the room, $H$, and the geometry of the vents - via the effective vent area, $A^{*}$. Purely natural ventilation is a convenient benchmark, against which to compare hybrid ventilation. For this reason, $Q_{N}$ and $g_{N}^{\prime}$ will be used throughout to scale volume fluxes and buoyancies, respectively.

A different scaling, using the mechanically-forced volume flux, $Q_{F}$, was considered. However, as the focus of the current study is to investigate the ventilation resulting from a range of forced volume flux settings, $Q_{F}$ will not be constant throughout our analysis and, hence, is inappropriate as a scaling factor. In contrast, the natural ventilation volume flux, $Q_{N}$, can be considered constant, as its components $\left(A^{*}, B\right.$ and $\left.H\right)$ are invariant throughout the theoretical analysis herein and describe well a given situation of a heated room and its relevant geometry.

\footnotetext{
${ }^{1}$ In the derivation of (7) the Bernouilli equation is applied at the venae contractae, which occur just downstream of the openings. This distance downstream is assumed to be small compared with the driving head producing the ventilation, so that $H$ is still the driving head. We consider sharp-edged openings (for which there are no frictional losses associated with the opening perimeter) and, as such, the use of 'loss' refers to the fractional reduction in area of the flow at the vena contracta relative to the opening.
} 


\section{$3 \quad$ Hybrid supply ventilation}

Consider a room with a constant heat input represented by the buoyancy flux $B$ and a forced supply volume flux $Q_{F}$, as illustrated schematically in figure $1 \mathrm{~b}$. Before developing mathematical expressions, some physical reasoning is applied in order to properly formulate the model.

If the fan volume flux is zero $\left(Q_{F}=0\right)$, a naturally ventilated room is established. For $Q_{F}>0$, a supply fan increases the static air pressure inside a ventilated room. Therefore, when comparing with the pressure difference distribution for purely natural ventilation (figure 2c), we expect the pressure difference distribution in hybrid supply ventilation to be shifted to the right. For sufficiently small values of $Q_{F}$, the direction of flow through the floor-level and ceiling-level vents will remain the same as that of a naturally ventilated room - inflow at floor level and outflow at ceiling level - this is referred to as 'forward' flow, and is addressed in $§ 3.1$.

As $Q_{F}$ is further increased, the air pressure at floor level within the room continues to increase. When the air pressure at floor level inside the room exceeds the air pressure at floor level outside the room, there will be outflow through the open floor-level vent, i.e. $Q_{1}$ will be negative - this is referred to as 'reverse' flow, addressed in $§ 3.2$.

At some critical value of the supply volume flux, herein referred to as $Q_{F, \text { crit }}$, the pressure difference at floor level, $\Delta p_{z=0}$, will be zero and, hence, no airflow is predicted through the floor-level vent.

In $\S 3.1$ and $\S 3.2$, expressions are developed for the inflow and outflow volume fluxes, the local buoyancy and the position of the neutral pressure level, in 'forward' and 'reverse' flow, respectively. We begin by considering forward flow.

\subsection{Hybrid supply, forward flow}

Figure 3a shows forward flow in a uniformly warm room with a supply fan providing an inflow volume flux, $Q_{F}$. Figure $3 \mathrm{~b}$ shows a schematic of the pressure difference distribution, $\Delta p(z)$. The thick (grey) line represents purely natural ventilation ( $c f$. figure $2 \mathrm{c}$ ), whilst the narrow (black) line represents hybrid supply ventilation. This schematic shows that the effect of a supply fan is to shift the static pressure difference distribution to the right, i.e. to increase the static pressure inside the room. Consequently, the neutral pressure level is shifted downwards and the pressure difference across the floor-level vent, $|\Delta p|_{z=0}$, decreases in magnitude, whilst the pressure difference across the ceilinglevel vent, $|\Delta p|_{z=H}$, increases.

Comparing the static pressure differences across the floor-level and ceiling-level vents, with the static pressure difference due to the buoyant warm air in the room, the balance of pressures can be expressed as

$$
|\Delta p|_{z=0}+|\Delta p|_{z=H}=\rho_{0} g^{\prime} H
$$

which is identical to (5), the pressure balance expression for purely natural ventilation. 


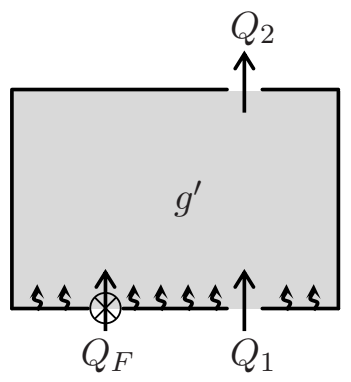

(a)

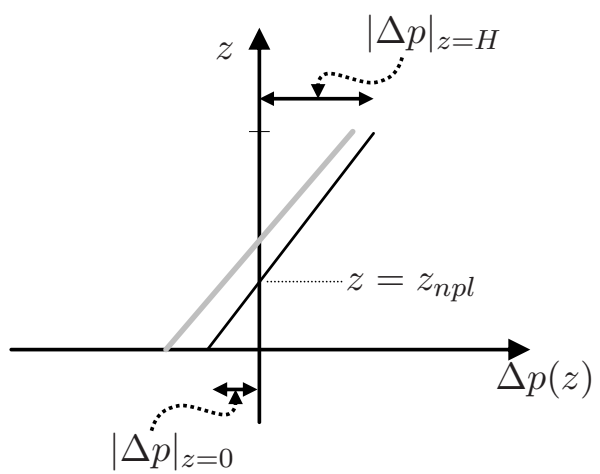

(b)

Figure 3: Schematic of forward flow with a supply fan showing: (a) a ventilated room; and (b) the corresponding vertical variation of static pressure difference, $\Delta p(z)$. In (b), the thick (grey) line is the pressure difference distribution from natural ventilation ( $c f$. figure $2 \mathrm{~b}$ ) and the narrower (black) line is the pressure difference distribution in forward flow, hybrid supply ventilation.

Assuming that the total ventilation volume flux through the space increases (which will be demonstrated later), the uniform reduced gravity of air within the room, $g^{\prime}$, will be lower in hybrid ventilation than would be expected in a naturally ventilated room subject to the same input buoyancy-flux. This explains the different gradients of the two pressure difference lines in figure $3 \mathrm{~b}$.

As in $\S 2$, the pressure difference terms in (11) can be replaced by the volume fluxes, $Q_{1}$ and $Q_{2}$, through those vents, leaving

$$
\frac{Q_{1}^{2}}{2 a_{1}^{2} c_{1}^{2}}+\frac{Q_{2}^{2}}{2 a_{2}^{2} c_{2}^{2}}=g^{\prime} H
$$

This expression relates the hybrid ventilation volume fluxes to the buoyancy within the room, and may be thought of as the hybrid equivalent of (6). If this were purely natural ventilation, we could write $Q_{1}=Q_{2}$ and, after introducing the effective vent area, $A^{*}$, derive (7), leading to (9). In hybrid ventilation, however, $Q_{1} \neq Q_{2}$. From inspection of figure $3 \mathrm{a}$, conservation of volume for an incompressible fluid requires that

$$
Q_{1}+Q_{F}=Q_{2} .
$$

\section{The floor-level inflow, $Q_{1}$}

In order to derive an expression for the floor-level inflow volume flux, $Q_{1}$, we substitute (13) into (12), to eliminate $Q_{2}$, leaving

$$
\frac{Q_{1}^{2}}{A^{* 2}}+\frac{Q_{F}^{2}}{2 a_{2}^{2} c_{2}^{2}}\left(1+2 \frac{Q_{1}}{Q_{F}}\right)=g^{\prime} H
$$


Multiplying through by $A^{* 2} / Q_{F}^{2}$, and recalling that $R^{*}=a_{1} c_{1} / a_{2} c_{2}$, so that

$$
\frac{A^{* 2}}{2 a_{2}^{2} c_{2}^{2}} \equiv \frac{R^{* 2}}{1+R^{* 2}}
$$

(14) becomes

$$
\left(\frac{Q_{1}}{Q_{F}}\right)^{2}+\left(\frac{R^{* 2}}{1+R^{* 2}}\right)\left(1+2 \frac{Q_{1}}{Q_{F}}\right)=\frac{A^{* 2} g^{\prime} H}{Q_{F}^{2}} .
$$

In a steady state, conservation of buoyancy requires that the buoyancy flux supplied by the heat source, $B$, is exactly balanced by the buoyancy flux lost via the outflow. This allows us to write $B=Q_{t o t} g^{\prime}$, which, with $Q_{t o t}=Q_{1}+Q_{F}$, can be rearranged to give

$$
g^{\prime}=\frac{B}{Q_{1}+Q_{F}} .
$$

Substituting (16) into (15), in order to eliminate $g^{\prime}$, leaves

$$
\begin{aligned}
\left(\frac{Q_{1}}{Q_{F}}\right)^{2}+\left(\frac{R^{* 2}}{1+R^{* 2}}\right)\left(1+2 \frac{Q_{1}}{Q_{F}}\right) & =\frac{A^{* 2} B H}{Q_{F}^{2}\left(Q_{1}+Q_{F}\right)} \\
& =\left(\frac{Q_{N}}{Q_{F}}\right)^{3}\left(1+\frac{Q_{1}}{Q_{F}}\right)^{-1}
\end{aligned}
$$

where we recall $Q_{N}=\left(A^{* 2} B H\right)^{1 / 3}$ is the steady volume flux for purely natural ventilation of an identical room. The floor-level volume flux, $Q_{1}$, is here expressed solely in terms of the supply fan volume flux, $Q_{F}$, the natural ventilation volume flux, $Q_{N}$, and the ratio of effective vent areas, $R^{*}$. With some algebra, (17) can be written in a convenient form as

$$
\left(\frac{Q_{N}}{Q_{F}}\right)^{3}=\left(1+\frac{Q_{1}}{Q_{F}}\right)\left[\left(\frac{Q_{1}}{Q_{F}}\right)^{2}+\left(\frac{R^{* 2}}{1+R^{* 2}}\right)\left(1+2 \frac{Q_{1}}{Q_{F}}\right)\right] .
$$

The quantity of interest in (18) is the floor-level inflow, $Q_{1} ;(18)$ is cubic in $Q_{1} / Q_{F}$. In order to solve for $Q_{1}$, the method used was to specify $R^{*}$ and $Q_{1} / Q_{F}$, and solve for $Q_{N} / Q_{F}$. After some manipulation, this provided $Q_{1} / Q_{N}$, in terms of $Q_{F} / Q_{N}$.

It is interesting that the effective vent area ratio, $R^{*}$, is a key parameter, in the form $R^{* 2} /\left(1+R^{* 2}\right)$, but the effective vent area, $A^{*}$, does not appear in (18). This is a feature of the chosen scaling on $Q_{N}$. Since $A^{*}, B$ and $H$ appear in $Q_{N}$, they are scaled out of and, hence, do not appear explicitly in (18); although they do appear implicitly, through $Q_{N}$.

\section{The ceiling-level outflow, $Q_{2}$}

Since the floor-level inflow volume flux is now known, $Q_{2}$ can be calculated by conservation of volume. However, we consider it informative to follow a similarly thorough derivation to that laid out above, allowing volume conservation to be used as a check of the final predictions. 
In order to develop an expression for the ceiling-level volume flux, $Q_{2}$, the volume conservation expression, (13), is rearranged, and substituted into (12). Thus, the floor-level volume flux, $Q_{1}$, is eliminated, leaving

$$
\frac{Q_{2}^{2}}{A^{* 2}}+\frac{Q_{F}^{2}}{2 a_{1}^{2} c_{1}^{2}}\left(1-2 \frac{Q_{2}}{Q_{F}}\right)=g^{\prime} H .
$$

Multiplying through by $A^{* 2} / Q_{F}^{2}$ gives

$$
\left(\frac{Q_{2}}{Q_{F}}\right)^{2}+\left(\frac{1}{1+R^{* 2}}\right)\left(1-2 \frac{Q_{2}}{Q_{F}}\right)=\frac{A^{* 2} g^{\prime} H}{Q_{F}^{2}},
$$

where

$$
\frac{1}{1+R^{* 2}} \equiv \frac{A^{* 2}}{2 a_{1}^{2} c_{1}^{2}} .
$$

Since $Q_{t o t}=Q_{2}$, conservation of buoyancy can be expressed simply as $g^{\prime}=B / Q_{2}$, which is substituted into (20) in order to eliminate the local buoyancy. This leaves

$$
\left(\frac{Q_{2}}{Q_{F}}\right)^{2}+\left(\frac{1}{1+R^{* 2}}\right)\left(1-2 \frac{Q_{2}}{Q_{F}}\right)=\frac{A^{* 2} B H}{Q_{F}^{2} Q_{2}}=\left(\frac{Q_{N}}{Q_{F}}\right)^{3}\left(\frac{Q_{F}}{Q_{2}}\right)
$$

wherein the ceiling-level volume flux is expressed solely in terms of the supply fan volume flux, the natural ventilation volume flux, and the ratio of effective vent areas. In the convenient form used throughout this paper, this becomes

$$
\left(\frac{Q_{N}}{Q_{F}}\right)^{3}=\left(\frac{Q_{2}}{Q_{F}}\right)\left[\left(\frac{Q_{2}}{Q_{F}}\right)^{2}+\left(\frac{1}{1+R^{* 2}}\right)\left(1-2 \frac{Q_{2}}{Q_{F}}\right)\right] .
$$

\section{The relative effectiveness of hybrid ventilation}

A measure is required by which one may assess the effectiveness of a hybrid ventilation strategy. One obvious metric is the extra ventilative cooling provided by the hybrid strategy when compared with purely natural ventilation. As mentioned previously, in ventilation it is convenient to consider the reduced gravity, or local buoyancy, in place of the temperature difference.

Herein, the uniform local buoyancy in hybrid ventilation is denoted $g^{\prime}$. The uniform local buoyancy resulting from purely natural ventilation of an identical room is $g_{N}^{\prime}$. The ratio $g^{\prime} / g_{N}^{\prime}$ expresses the relative dilution of buoyancy in hybrid ventilation, compared to purely natural ventilation, thereby quantifying the extra ventilative cooling provided by the hybrid strategy. Values of $g^{\prime} / g_{N}^{\prime}<1$ indicate greater dilution of buoyancy (i.e. a cooler room) in hybrid ventilation than natural ventilation. This provides an objective metric by which to compare the relative effectiveness of the hybrid strategy.

We know from (1) that, regardless of the ventilation strategy, conservation of buoyancy requires $B=Q_{t o t} g^{\prime}$. In purely natural ventilation, the total ventilation volume flux is the natural ventilation volume 
flux, i.e. $Q_{t o t}=Q_{N}$. As the natural and hybrid systems are subject to the same input buoyancy flux, it follows that

$$
\frac{g^{\prime}}{g_{N}^{\prime}}=\frac{Q_{N}}{Q_{t o t}} .
$$

In forward flow hybrid supply ventilation, the total ventilation volume flux is equal to the ceiling-level outflow volume flux, $Q_{2}$. Thus, (23) becomes

$$
\frac{g^{\prime}}{g_{N}^{\prime}}=\frac{Q_{N}}{Q_{2}},
$$

where $g^{\prime} / g_{N}^{\prime}$ can be thought of as a 'ventilation effectiveness ratio'.

\section{The neutral pressure level, $z_{n p l}$}

The final quantity of interest is the neutral pressure level, $z_{n p l}$. We recap that the neutral pressure level is a horizontal plane over which the air pressure inside and outside a ventilated room are equal $\left(\Delta p\left(z_{n p l}\right)=0\right)$. In buoyancy-driven ventilation, the pressure difference across an open vent is determined by the net buoyancy between the horizontal plane of the vent and the neutral pressure level. This allows us to write

$$
\frac{Q_{1}^{2}}{2 a_{1}^{2} c_{1}^{2}}=g^{\prime} z_{n p l} .
$$

Dividing through by $g^{\prime} H$ and introducing the effective vent area, $A^{*}$, the normalised height of the neutral pressure level above the floor can be expressed as

$$
\frac{z_{n p l}}{H}=\frac{Q_{1}^{2}}{A^{* 2} g^{\prime} H}\left(\frac{1}{1+R^{* 2}}\right) .
$$

With $Q_{t o t}=Q_{1}+Q_{F}$, conservation of buoyancy can be expressed as $g^{\prime}=B /\left(Q_{1}+Q_{F}\right)$, which can be substituted into (26). Thus, the position of the neutral pressure level can be expressed in terms of the floor-level inflow volume flux, the forced supply volume flux, the natural ventilation volume flux and the effective vent area ratio:

$$
\begin{aligned}
\frac{z_{n p l}}{H} & =\frac{Q_{1}^{2}\left(Q_{1}+Q_{F}\right)}{A^{* 2} B H}\left(\frac{1}{1+R^{* 2}}\right) \\
& =\left(\frac{Q_{1}}{Q_{N}}\right)^{3}\left(1+\frac{Q_{F}}{Q_{1}}\right)\left(\frac{1}{1+R^{* 2}}\right) .
\end{aligned}
$$

Since the inflow volume flux, $Q_{1}$, is itself solely a function of $Q_{F}, Q_{N}$ and $R^{*}$ (see (18)), $z_{n p l} / H$ is, in effect, a function of only these three quantities.

So far in this section, expressions have been developed which describe the floor- and ceiling-level volume fluxes, the local buoyancy and the position of the neutral pressure level, in forward flow, hybrid supply ventilation. These expressions, (18), (22), (24) and (26), respectively, are summarised in table 1 , which also records the volume conservation 


$$
Q_{1}+Q_{F}=Q_{2}
$$

Pressure

$$
|\Delta p|_{z=0}+|\Delta p|_{z=H}=\rho_{0} g^{\prime} H
$$

$$
\begin{gathered}
Q_{1}\left(\frac{Q_{N}}{Q_{F}}\right)^{3}=\left(1+\frac{Q_{1}}{Q_{F}}\right)\left[\left(\frac{Q_{1}}{Q_{F}}\right)^{2}+\left(\frac{R^{* 2}}{1+R^{* 2}}\right)\left(1+2 \frac{Q_{1}}{Q_{F}}\right)\right] \\
Q_{2} \quad\left(\frac{Q_{N}}{Q_{F}}\right)^{3}=\left(\frac{Q_{2}}{Q_{F}}\right)\left[\left(\frac{Q_{2}}{Q_{F}}\right)^{2}+\left(\frac{1}{1+R^{* 2}}\right)\left(1-2 \frac{Q_{2}}{Q_{F}}\right)\right] \\
g^{\prime} \quad \frac{g^{\prime}}{g_{N}^{\prime}}=\frac{Q_{N}}{Q_{2}} \\
\frac{z_{n p l}}{H}=\left(\frac{Q_{1}}{Q_{N}}\right)^{3}\left(1+\frac{Q_{F}}{Q_{1}}\right)\left(\frac{1}{1+R^{* 2}}\right)
\end{gathered}
$$

Table 1: Summary of expressions for forward flow, hybrid supply ventilation

expression, (13), and the pressure balance relationship, (11). Solutions of these expressions are plotted in figures $4-7$. Fans generate their own pressure difference that depends on the fan speed and geometry. As such, for the configuration considered, the flow will adjust along the solution curves presented to match the fan performance curve.

In $\S 3.2$, we consider reverse flow, hybrid supply ventilation, in which the volume flux through the open floor-level vent is outflow.

\subsection{Hybrid supply, reverse flow}

For reverse flow, the mathematical development is very similar to that of forward flow, as laid out in detail above. For this reason, it is not considered worthwhile herein to describe each step in full detail; the complete model development is described by Connick (2013). Herein, the key results of primary interest are summarised in table 2 . These results are plotted in figures $4-7$ in $§ 3.4$. The solid lines depict the full physical solution, achieved by plotting the relevant two component solutions (for forward flow and reverse flow) in the ranges over which they are valid physically. The dashed lines show the non-physical theoretical extensions of the component solutions and are retained as they indicate how the full physical solution is formed from the underlying polynomial components. 


$$
\begin{aligned}
& Q_{1} \quad\left(\frac{Q_{N}}{Q_{F}}\right)^{3}=\left(\frac{1}{1+R^{* 2}}\right)\left[\left(R^{* 2}-1\right)\left(\frac{Q_{1}}{Q_{F}}\right)^{2}+R^{* 2}\left(1-2 \frac{Q_{1}}{Q_{F}}\right)\right] \\
& Q_{2} \quad\left(\frac{Q_{N}}{Q_{F}}\right)^{3}=\left(\frac{1}{1+R^{* 2}}\right)\left[\left(\frac{Q_{2}}{Q_{F}}\right)^{2}\left(R^{* 2}-1\right)-\left(1-2 \frac{Q_{2}}{Q_{F}}\right)\right] \\
& g^{\prime} \quad \frac{g^{\prime}}{g_{N}^{\prime}}=\frac{Q_{N}}{Q_{F}} \\
& z_{n p l} \quad \frac{z_{n p l}}{H}(\text { virtual })=-\left(\frac{Q_{1}}{Q_{N}}\right)^{3}\left(\frac{Q_{F}}{Q_{1}}\right)\left(\frac{1}{1+R^{* 2}}\right)
\end{aligned}
$$

Table 2: Summary of expressions for reverse flow, hybrid supply ventilation. (The modulus of $Q_{1}$ is taken to remove any ambiguity associated with $Q_{1}$ being (negative) outflow.)

\subsection{The critical supply volume flux}

The critical value of $Q_{F}$, herein denoted $Q_{F, \text { crit }}$, marks the transition between forward flow and reverse flow. At this critical supply volume flux the pressure difference across and, hence, the volume flux through the floor-level vent is predicted to be zero. Thus, setting $Q_{1}=0$ in either (18) or (36), we find that

$$
\frac{Q_{F, c r i t}}{Q_{N}}=\left(\frac{1+R^{* 2}}{R^{* 2}}\right)^{1 / 3} .
$$

The critical supply volume flux is illustrated in figures 4 to 7 by asterisks on the $Q_{F} / Q_{N}$ axis.

Recalling that $R^{*}=a_{1} c_{1} / a_{2} c_{2}$, we note that increasing the effective vent area ratio decreases $Q_{F, \text { crit }} ;$ i.e. if the floor-level vents are large compared to the ceiling-level vents, reverse flow is expected at relatively small values of $Q_{F}$.

This can be explained by considering an alternative (although equivalent) definition of $Q_{F, \text { crit }}$; namely the forced supply volume flux for which the neutral pressure level is at floor level, $z_{n p l} / H=0$. Regardless of the type of ventilation (purely natural, hybrid supply or hybrid extract), larger values of $R^{*}$ result in a neutral pressure level closer to the floor. By this reasoning, for larger values of $R^{*}$ in hybrid supply ventilation, $z_{n p l} / H=0$ will occur at smaller values of $Q_{F} / Q_{N}$. 
This analysis and reasoning allows us to conclude that, in hybrid supply ventilation, in order to make reverse flow less likely, the value of $R^{*}$ should be small; i.e. the floor-level vents should be small relative to the ceiling-level vents.

\subsection{Hybrid supply ventilation - discussion of re- sults}

Mathematical models have been developed [in $§ 3.1-\S 3.3]$ to describe hybrid supply ventilation of an isolated room. Specifically, expressions have been developed for the volume fluxes through floor-level and ceiling-level vents, $Q_{1}$ and $Q_{2}$, respectively, the uniform local buoyancy, $g^{\prime}$, which represents the temperature of air within the room relative to the exterior, and the position (the height above the floor) of the neutral pressure level, $z_{n p l}$.

These solutions are now plotted in order to illustrate the effect of imposing a mechanically forced volume flux in an otherwise naturally ventilated room. For example, a ventilation design engineer may be interested in how much cooler the room will be in hybrid rather than purely natural ventilation, or what the effect might be of doubling the supply fan volume flux, $Q_{F}$ - does this halve the room temperature? And although reverse flow is generally assumed to be undesirable, what are the main differences between forward and reverse flow in terms of the total ventilation?

It is important to realise that each expression developed herein is only valid under the conditions (flow direction) for which it was derived. Expressions that describe forward flow are not valid if $Q_{F}>Q_{F, \text { crit }}$. Similarly, expressions that describe reverse flow are not valid for $Q_{F}<Q_{F, \text { crit }}$. In figures $4-7$, solid lines represent the physical solution and dashed lines represent the continuation of the mathematical solutions. Dashed lines are provided for illustrative purposes only and do not represent physical solutions.

Each figure shows three lines, representing solutions with $R^{*}=0.5$, $R^{*}=1$ and $R^{*}=2$; increasing line thicknesses representing larger $R^{*}$. These values of the effective vent area ratio were chosen to span, what is considered to be, a reasonable range of vent areas in a typical room. From a practical perspective, floor-level vents which are more than twice, or less than half, the area of the ceiling-level vents (in the same room) are uncommon.

All figures in this section show some quantity of interest plotted as a function of the forced supply volume flux, $Q_{F}$, scaled on the natural ventilation volume flux, $Q_{N}$. This scaling, $Q_{N}=\left(A^{* 2} B H\right)^{1 / 3}$, is a function of the source buoyancy flux, $B$, and the geometry of the room, through $A^{*}$ and $H$. It is convenient to scale volume fluxes on $Q_{N}$, as this generalises the results shown, and provides a contextual comparison for the magnitude of the forced supply volume flux, which would not be possible using unscaled values. In order to interpret the figures, one should consider that $Q_{N}$ is a constant, although no value of $Q_{N}$ has been specified in order to plot these figures. The convenience of 
representing the results in this general, non-dimensional form is that, if numerical values of the results are required, one need only multiply the non-dimensional results by the value of $Q_{N}$ which is of interest.

\section{Effect on floor-level and ceiling-level volume fluxes}

Figures 4 and 5 show the variation of the volume flux through floorlevel and ceiling-level vents, respectively, in hybrid supply ventilation. These figures illustrate the effect of increasing the forced supply volume flux, $Q_{F}$, on the variable volume fluxes, $Q_{1}$ and $Q_{2}$, through the open vents.

In figure $4, Q_{1} / Q_{N}$ is plotted as a function of $Q_{F} / Q_{N}$ for three values of $R^{*}$. In other words, we determine how $Q_{1}$ varies with the strength of the hybrid forcing $Q_{F}$. Figure 4 shows that the effect of imposing a forced supply volume flux is to decrease the floor-level inflow volume flux, $Q_{1}$. For small forced volume flux settings, the natural ventilation solution is recovered (i.e. as $Q_{F} / Q_{N} \rightarrow 0, Q_{1} / Q_{N} \rightarrow 1$ ). As the fan volume flux is increased, $Q_{1} / Q_{N}$ decreases monotonically for all $Q_{F} / Q_{N}$. Asterisks mark the value of $Q_{F, c r i t}$, as defined in (40). For $Q_{F}<Q_{F, c r i t}$, the physical solution (solid lines) is the solution of (18). For $Q_{F}>Q_{F, \text { crit }}$, the physical solution follows the reverse flow expression, (36).

By comparing the different lines in figure 4 , it can be seen that, for $R^{*}=0.5$, the rate of change of $Q_{1} / Q_{N}$ with $Q_{F} / Q_{N}$ is less than for $R^{*}=2$. Furthermore, the critical forced volume flux, $Q_{F, \text { crit }}$, beyond which the flow through the floor-level vent reverses, is larger for smaller values of $R^{*}$. As a consequence, in hybrid supply ventilation, if reduction and reversal of the airflow through the floor-level vent is undesirable, it is better to design floor-level vents which are smaller than the ceiling-level vents $\left(R^{*}<1\right)$.

For sufficiently large values of $Q_{F} / Q_{N}$, in the reverse flow region, the variation of $Q_{1} / Q_{N}$ with $Q_{F} / Q_{N}$ tends towards a constant gradient, which depends upon the effective vent area ratio, $R^{*}$.

Figure 5 shows the ceiling-level volume flux, $Q_{2} / Q_{N}$, plotted as a function of the forced supply volume flux, $Q_{F} / Q_{N}$. For small values of the forced supply volume flux, $Q_{F}<Q_{F, \text { crit }}$, the physical solution (solid lines) is the solution of (22). For $Q_{F}>Q_{F, c r i t}$, the physical solution is the solution of (37). The critical forced supply volume flux is marked by an asterisk and can be clearly identified in figure 5 by an abrupt change in the gradient of the physical solution.

By following the $R^{*}=2$ line in figure 5 , it can be seen that increasing $Q_{F} / Q_{N}$ from $Q_{F} / Q_{N}=0$ to $Q_{F} / Q_{N}=1$ achieves only a minor increase in $Q_{2} / Q_{N}$, whilst for $R^{*}=0.5$ a much greater increase in $Q_{2} / Q_{N}$ is shown over this same range. This behaviour can be justified by considering the effect of a supply fan on the position of the neutral pressure level. A supply fan increases the air pressure inside a ventilated room and, hence, causes the neutral pressure level to move downwards $\left(z_{n p l} / H \downarrow\right)$. For $R^{*}=0.5$, the neutral pressure level in natural ventilation is relatively close to the ceiling; when a forced supply, 


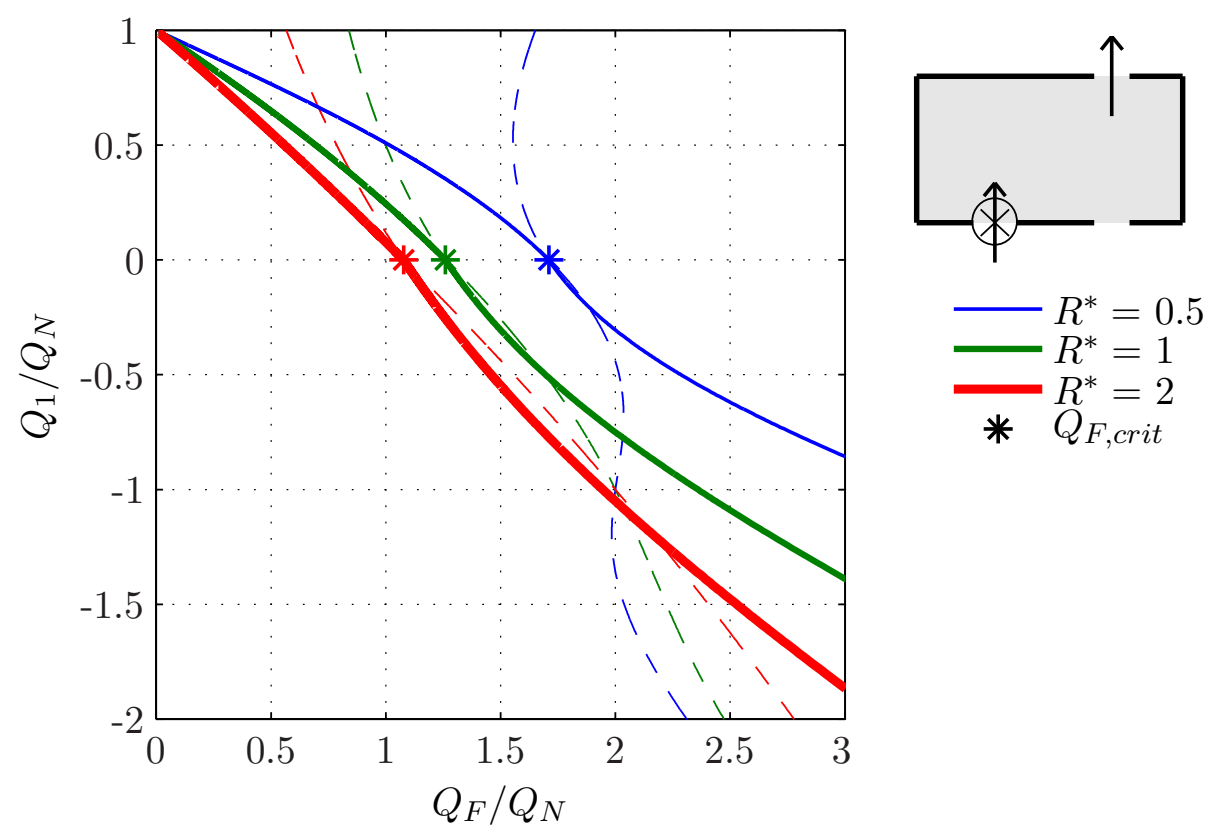

Figure 4: Hybrid supply ventilation. The floor-level volume flux, $Q_{1} / Q_{N}$, plotted as a function of forced supply volume flux, $Q_{F} / Q_{N}$, for three values of the effective vent area ratio, $R^{*}$. Asterisks mark the critical supply volume flux, $Q_{F}=Q_{F, \text { crit }}$. For $Q_{F}>Q_{F, \text { crit }}$, the floor-level volume flux, $Q_{1}<0$; this is known as reverse flow.

$Q_{F}$, is added, this tends to move the neutral pressure level downwards, which has a significant effect on $Q_{2}$. Conversely, for $R^{*}=2$, the neutral pressure level in natural ventilation is close to the floor; when a forced supply volume flux is added (or increased), the effect on $Q_{2}$ of the neutral pressure level moving downward is less pronounced, as the neutral pressure level is nearer the floor to begin with. Analysis of the neutral pressure level will be revisited in the discussion of figure 7 .

In contrast to figure 4, which showed the floor-level volume flux $Q_{1}$, figure 5 shows that the ceiling-level volume flux $Q_{2}$ is not monotonic for the values of the effective vent area shown. The $R^{*}=1$ line, for example, clearly shows that, when the physical solution (solid line) switches from forward to reverse flow, there is a decrease in $Q_{2} / Q_{N}$ and a local minimum, before $Q_{2} / Q_{N}$ recovers. This results in there being multiple values of $Q_{F} / Q_{N}$ which may result in identical values of $Q_{2} / Q_{N}$. For example, following the $R^{*}=1$ line, $Q_{2} / Q_{N} \approx 1.2$ can be achieved with $Q_{F} / Q_{N} \approx\{1.0,1.3$ or 1.9$\}$. If the aim of providing a supply fan was to increase the airflow rate through the ceiling-level vent, this (multi-valued) solution demonstrates that one might put a great deal of energy into increasing $Q_{F}$, for very little or no gain in terms of the airflow rate $Q_{2}$, through the ceiling-level vent. 


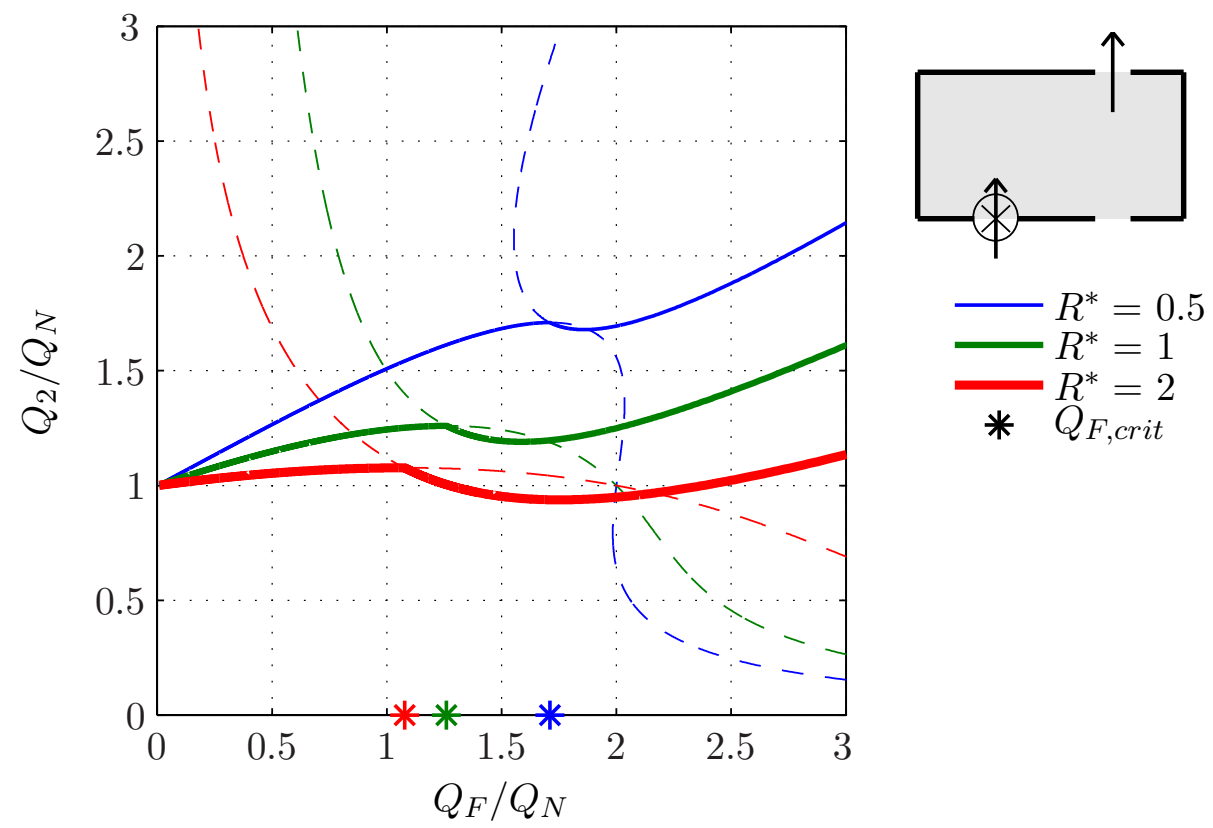

Figure 5: Hybrid supply ventilation. The ceiling-level volume flux, $Q_{2} / Q_{N}$, plotted as a function of forced supply volume flux, $Q_{F} / Q_{N}$, for three values of the effective vent area ratio, $R^{*}$.

For $R^{*}=2$, we predict that, in the region $Q_{F} / Q_{N} \approx 1.5$, the outflow through the ceiling-level vent drops below its natural ventilation value, i.e. $Q_{2} / Q_{N}<1$. The region where $Q_{2} / Q_{N}$ locally decreases is in the reverse flow regime, wherein the floor-level volume flux, $Q_{1}$, is outflow. In this region, the total volume flux through the room, $Q_{t o t}$, continues to increase, despite the local decrease in $Q_{2}$.

For large $Q_{F} / Q_{N}$ the solutions continue their upwards trends, eventually tending to a constant gradient, which depends on the effective vent area ratio.

\section{Effect on local buoyancy}

Figure 6 shows the variation of the scaled local buoyancy, $g^{\prime} / g_{N}^{\prime}$, with forced supply volume flux, $Q_{F} / Q_{N}$, for the same three values of the effective vent area ratio, $R^{*}$. This quantifies the relative effectiveness of a hybrid ventilation strategy, when compared to purely natural ventilation. Similarly to figures 4 and 5 , as $Q_{F} / Q_{N} \rightarrow 0$, the natural ventilation solution, in this case $g^{\prime} / g_{N}^{\prime} \rightarrow 1$, is recovered. For $Q_{F}<Q_{F, \text { crit }}$, there is an implied dependence on $R^{*}$. This is due to the presence of $Q_{2}$ in (24), as $Q_{2}$ is a function of $R^{*}$. For $Q_{F}>Q_{F, c r i t}$, however, the three plotted lines are coincident - in reverse flow, $Q_{t o t}=Q_{F}$ and, hence, $g^{\prime} / g_{N}^{\prime}$ is independent of $R^{*}$ (as described by (38)).

This figure provides perhaps the most obvious and compelling evi- 


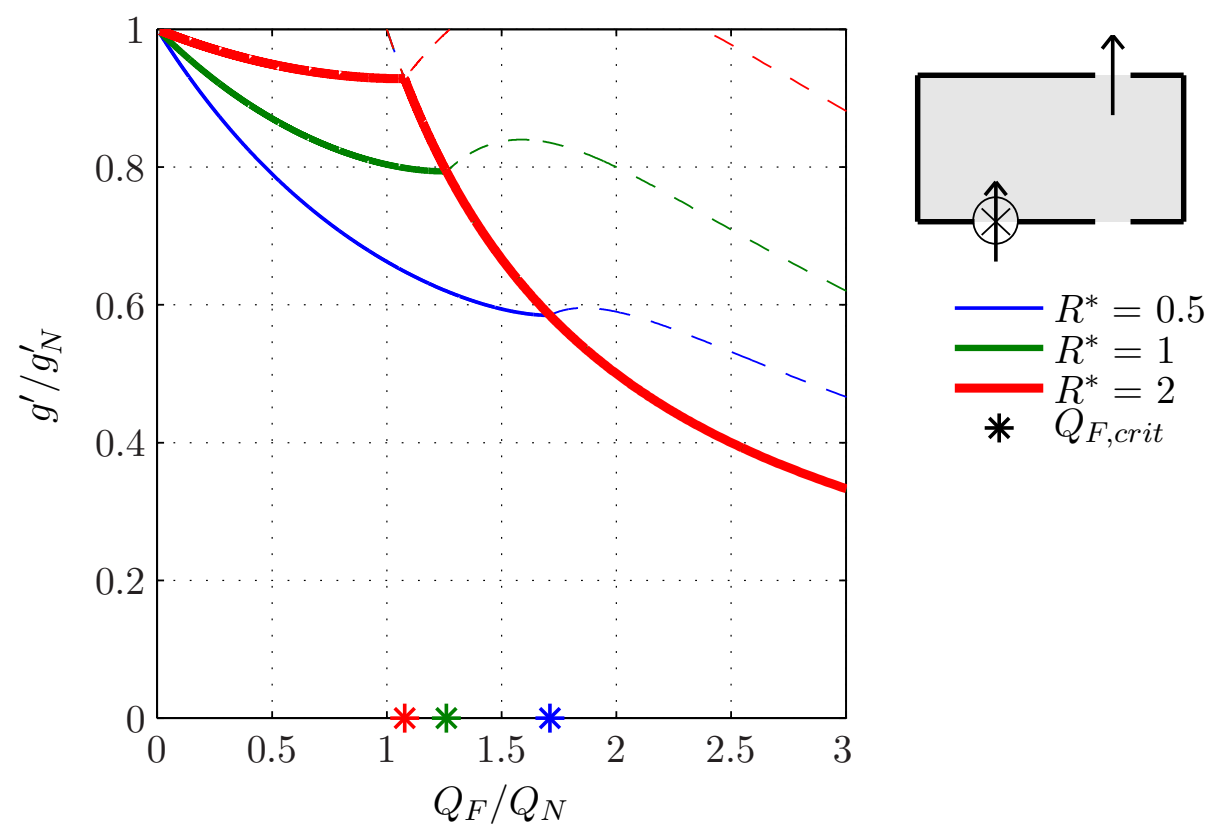

Figure 6: Hybrid supply ventilation. The steady local buoyancy, $g^{\prime} / g_{N}^{\prime}$, plotted as a function of forced supply volume flux, $Q_{F} / Q_{N}$, for three values of the effective vent area ratio, $R^{*}$.

dence for the wisdom of designing for small $R^{*}$, if one intends to employ a supply fan. For $R^{*}=2$, the effect of increasing $Q_{F} / Q_{N}$ from 0 to 1 produces only a small reduction in $g^{\prime} / g_{N}^{\prime}$. In contrast, for $R^{*}=0.5$, the same increase in $Q_{F} / Q_{N}$ results in a far greater reduction in $g^{\prime} / g_{N}^{\prime}$, corresponding to more effective cooling of the room.

\section{Effect on neutral pressure level}

The final figure in this hybrid supply section, figure 7 , plots $z_{n p l} / H$ against $Q_{F} / Q_{N}$. The figure shows how the neutral pressure level descends as the forced supply volume flux is increased. Similar to the previous figures, thick solid lines represent physically valid solutions, whilst the thin dashed lines represent the continuation of non-physical mathematical solutions.

It has been emphasised previously that the position of the neutral pressure level is a useful and intuitive tool for (physically) understanding the ventilation airflow in a room.

In figure 7 , the solutions at $Q_{F} / Q_{N}=0$ represent the position of the neutral pressure level in natural ventilation; for $R^{*}=\{0.5,1,2\}$, $\left(z_{n p l} / H\right)_{N}=\{0.8,0.5,0.2\}$, respectively. Hybrid ventilation with $Q_{F} / Q_{N}=0$ is purely natural ventilation. This figure shows that a unit increase in $Q_{F} / Q_{N}$ has a larger impact on $z_{n p l} / H$ for smaller values of $R^{*}$. 


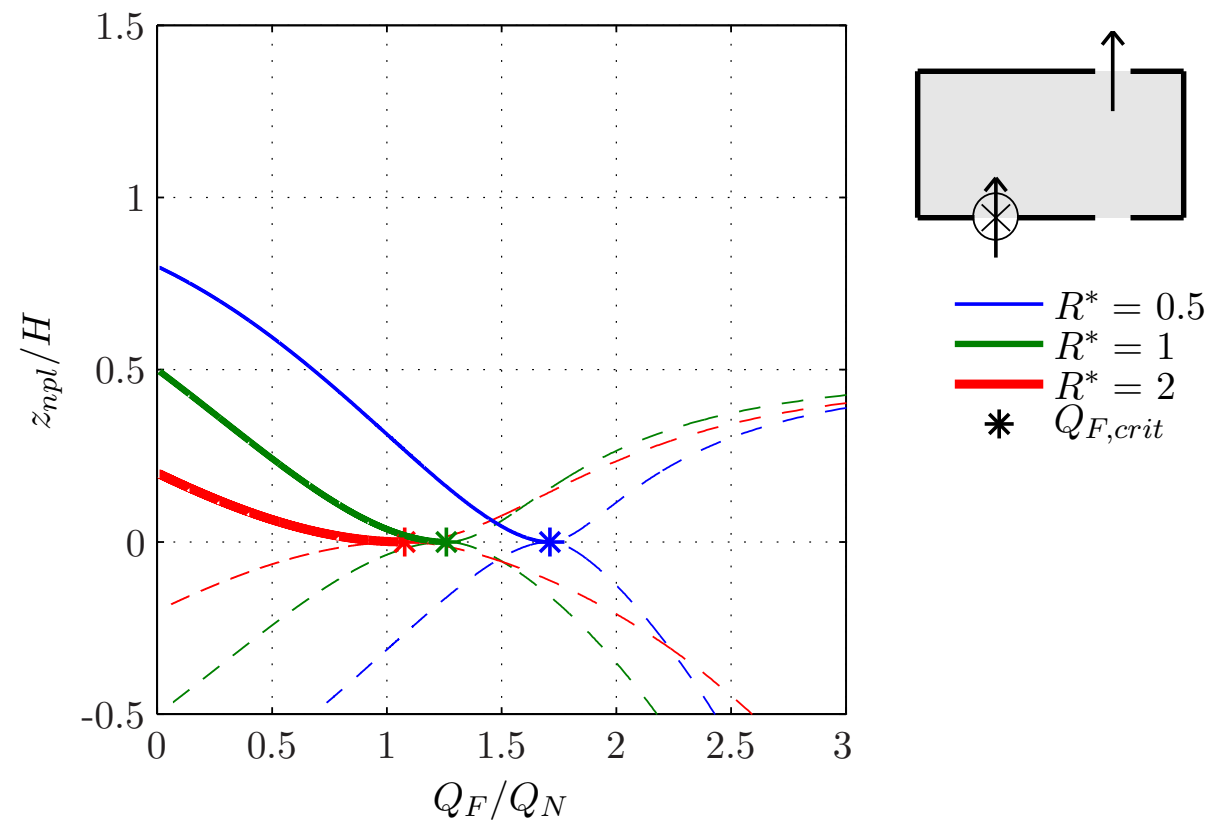

Figure 7: Hybrid supply ventilation. The position of the neutral pressure level, $z_{n p l} / H$, plotted as a function of forced supply volume flux, $Q_{F} / Q_{N}$, for three values of the effective vent area ratio, $R^{*}$. Dashed lines in the region $z_{n p l} / H>0$ are provided to illustrate the mathematical solution of (27). Dashed lines in the region $z_{n p l} / H<0$ are the reverse flow solution (39).

In the region $z_{n p l} / H<0$ in figure 7 , the dashed lines are solutions of (39), which express the position of the virtual neutral pressure level, in reverse flow. Whilst there is no physical neutral pressure level in reverse flow, these solutions provide some measure of what reduction in $Q_{F} / Q_{N}$ would be required in order to return to forward flow.

\subsection{Hybrid supply ventilation - summary}

To summarise this section on hybrid supply ventilation:

- the effect of a forced supply volume flux is to increase the air pressure within a ventilated room;

- this results in the neutral pressure level moving downwards, i.e. towards the floor.

The effect of increasing $Q_{F} / Q_{N}$ in hybrid supply ventilation is:

- $Q_{1} / Q_{N}$ decreases monotonically, eventually tending to a constant gradient which depends on $R^{*}$;

- $Q_{2} / Q_{N}$ generally increases, but experiences local minima, also eventually tending to a constant gradient; 
- $g^{\prime} / g_{N}^{\prime}$ decreases, and for $Q_{F}>Q_{F, c r i t}$ the solution is independent of $R^{*}$;

- these effects are greater if $R^{*}$ is small.

\section{Hybrid extract ventilation}

The focus of the study now moves to hybrid extract ventilation in which a predominantly buoyancy-driven ventilation strategy is augmented by a mechanical extract fan.

In a similar format to $\S 3$, the analysis begins with some physical reasoning to guide the formulation of the mathematical model. The mathematical development of key quantities in hybrid extract ventilation is very similar to that laid out above. For this reason, it is not deemed worthwhile to describe each step of the model development in full detail; it is the final results which are of primary interest.

Forward flow is again defined as flow in the same sense as that driven by the buoyancy alone - inflow through the floor-level vent and outflow through the ceiling-level vent. In contrast to the previous section, the criterion for reverse flow here, with an extract fan, is reversal of the ceiling-level volume flux, $Q_{2}$. For sufficiently small values of the forced extract volume flux $Q_{F}$, the ceiling-level volume flux will be outflow. As the extract fan volume flux is increased, the air pressure within the room decreases and the pressure difference across the ceiling-level vent also decreases. At a critical value of the forced extract volume flux, $Q_{F}=Q_{F, \text { crit }}$, the pressure difference across and, hence, the predicted volume flux through the ceiling-level vent will be zero. For all values of the forced extract volume flux which exceed this critical value, the volume flux through the ceiling-level vent will be negative, $Q_{2}<0$, signifying inflow.

Forward flow is considered first, in $\S 4.1$, followed by reverse flow in $\S 4.2$. The value of $Q_{F, c r i t}$ in hybrid extract ventilation is established in $\S 4.3$. The predictions are plotted and discussed in $\S 4.4$.

\subsection{Hybrid extract, forward flow}

The key results, for forward flow hybrid extract ventilation, are summarised in table 3 .

We note that (42) is identical to (5) and (11). The expression which describes the balance of pressures in forward flow hybrid ventilation, whether supply or extract, is identical to the pressure balance expression in purely natural ventilation.

\subsection{Hybrid extract, reverse flow}

The key results in reverse flow, hybrid extract ventilation, are summarised in table 4.

Unlike the forward flow expressions, the pressure balance expressions in reverse flow differ between supply and extract ventilation. 


\begin{tabular}{|c|c|c|}
\hline Flow rates & $Q_{1}=Q_{2}+Q_{F}$ & $(41)$ \\
\hline Pressure & $|\Delta p|_{z=0}+|\Delta p|_{z=H}=\rho_{0} g^{\prime} H$ & $(42)$ \\
\hline$Q_{1}$ & $\left(\frac{Q_{N}}{Q_{F}}\right)^{3}=\left(\frac{Q_{1}}{Q_{F}}\right)\left[\left(\frac{Q_{1}}{Q_{F}}\right)^{2}+\left(\frac{R^{* 2}}{1+R^{* 2}}\right)\left(1-2 \frac{Q_{1}}{Q_{F}}\right)\right]$ & (43) \\
\hline$Q_{2}$ & $\left(\frac{Q_{N}}{Q_{F}}\right)^{3}=\left(1+\frac{Q_{2}}{Q_{F}}\right)\left[\left(\frac{Q_{2}}{Q_{F}}\right)^{2}+\left(\frac{1}{1+R^{* 2}}\right)\left(1+2 \frac{Q_{2}}{Q_{F}}\right)\right]$ & $(44)$ \\
\hline$g^{\prime}$ & $\frac{g^{\prime}}{g_{N}^{\prime}}=\frac{Q_{N}}{Q_{1}}$ & $(45)$ \\
\hline$z_{n p l}$ & $\frac{z_{n p l}}{H}=\left(\frac{Q_{1}}{Q_{N}}\right)^{3}\left(\frac{1}{1+R^{* 2}}\right)$ & $(46)$ \\
\hline
\end{tabular}

Table 3: Summary of expressions for forward flow, hybrid extract ventilation

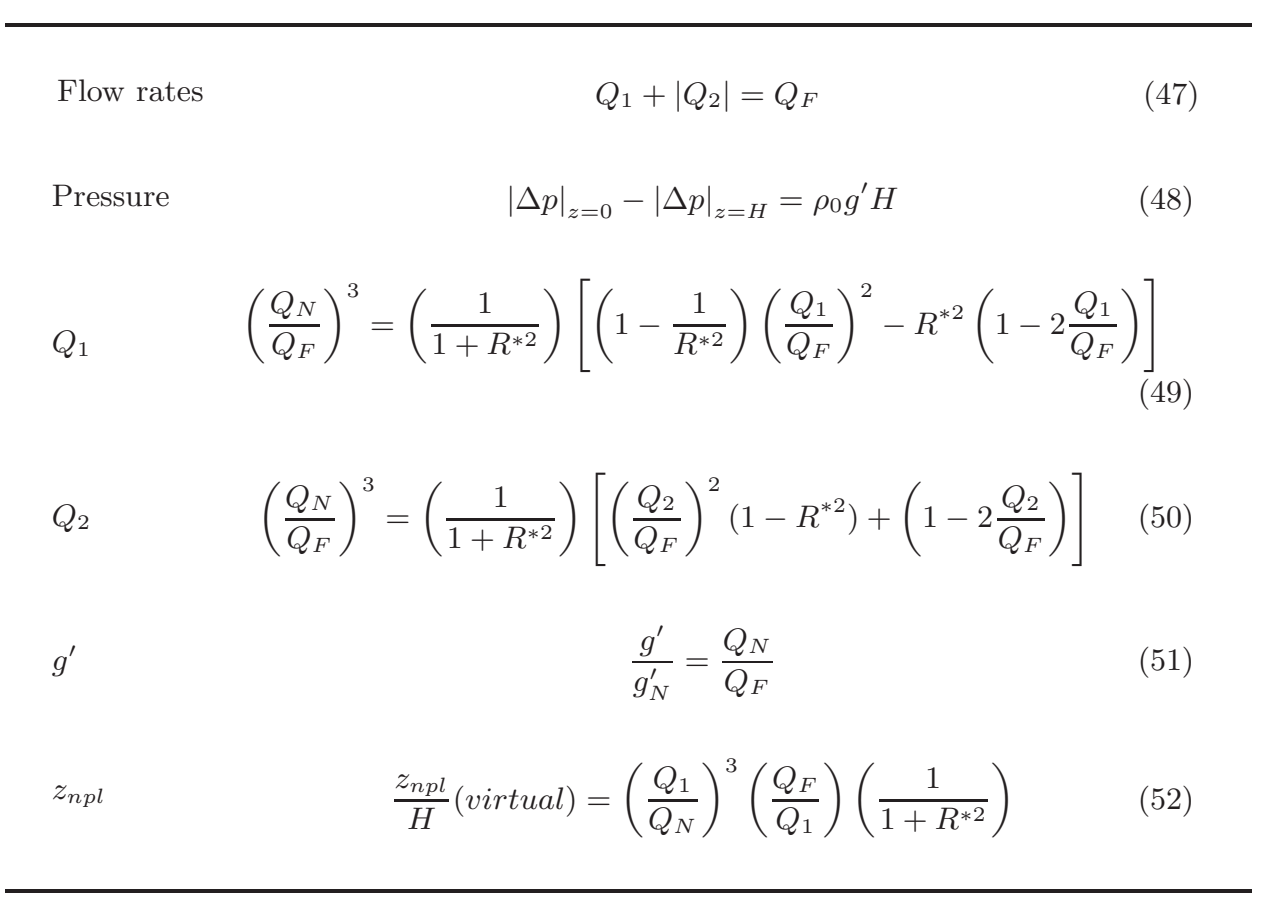

Table 4: Summary of expressions for reverse flow, hybrid extract ventilation 


\subsection{The critical extract volume flux}

The critical extract volume flux $Q_{F, \text { crit }}$ marks the boundary between forward and reverse flow. Setting $Q_{2}=0$ in either (44) or (50) gives

$$
\frac{Q_{F, c r i t}}{Q_{N}}=\left(1+R^{* 2}\right)^{1 / 3} \text {. }
$$

This expression contrasts with $Q_{F, \text { crit }}$ in hybrid supply ventilation, (40), wherein smaller values of $R^{*}$ resulted in larger values of $Q_{F, \text { crit }}$.

\subsection{Hybrid extract ventilation - discussion of re- sults}

The mathematical expressions in table 3 and table 4 describe the hybrid extract ventilation of a room. Solutions to these mathematical expressions exist over a wide domain, however, their physical validity is limited. In the following figures $(8-11)$, thick, solid lines represent the physical solution and thin, dashed lines represent the continuation of mathematical solutions. Where dashed lines are plotted, their solutions are not physically valid; the dashed lines are provided only to illustrate the continuation of the mathematical solution.

Figure 8, plots $Q_{1} / Q_{N}$ against $Q_{F} / Q_{N}$ for hybrid extract ventilation. On inspection, this figure is very similar in appearance to figure 5, which shows the variation of $Q_{2} / Q_{N}$ with $Q_{F} / Q_{N}$ in hybrid supply ventilation.

Indeed, the $R^{*}=1$ lines in figure 8 and figure 5 are identical and, by comparing the relevant expressions, it can be shown that for $R^{*}=1$, the supply fan solutions for $Q_{2} / Q_{N},(22)$ and (37), are identical to the extract fan solutions for $Q_{1} / Q_{N},(43)$ and (49), respectively. This implies that, for $R^{*}=1$, the effect of an extract fan on the floor-level volume flux is identical to the effect of a supply fan on the ceiling-level volume flux.

In figure $9, Q_{2} / Q_{N}$, is plotted as a function of the extract volume flux, $Q_{F} / Q_{N}$. As in the previous discussion, we can identify similarities between figure 9 and figure 4 , where figure 4 shows the variation of $Q_{1} / Q_{N}$ in hybrid supply ventilation.

Physical justification for this behaviour can be found by considering the pressure variation within a ventilated room. The effect of a supply fan is to increase the pressure within a room, thereby shifting the static pressure difference distribution to the right, when compared to that of a naturally ventilated room - as shown in figure $3 \mathrm{~b}$. Conversely, the effect of an extract fan is to decrease the pressure within a ventilated room, thereby shifting the natural pressure difference distribution to the left.

The direction in which the pressure difference distribution is shifted depends on the direction (supply or extract) of the forced mechanical volume flux $Q_{F}$, but the magnitude of this shift depends only on the magnitude of $Q_{F} / Q_{N}$. A given value of $Q_{F} / Q_{N}$ in hybrid supply ventilation reduces the pressure difference across the floor-level vent by a set magnitude. The same value of $Q_{F} / Q_{N}$ in hybrid extract 


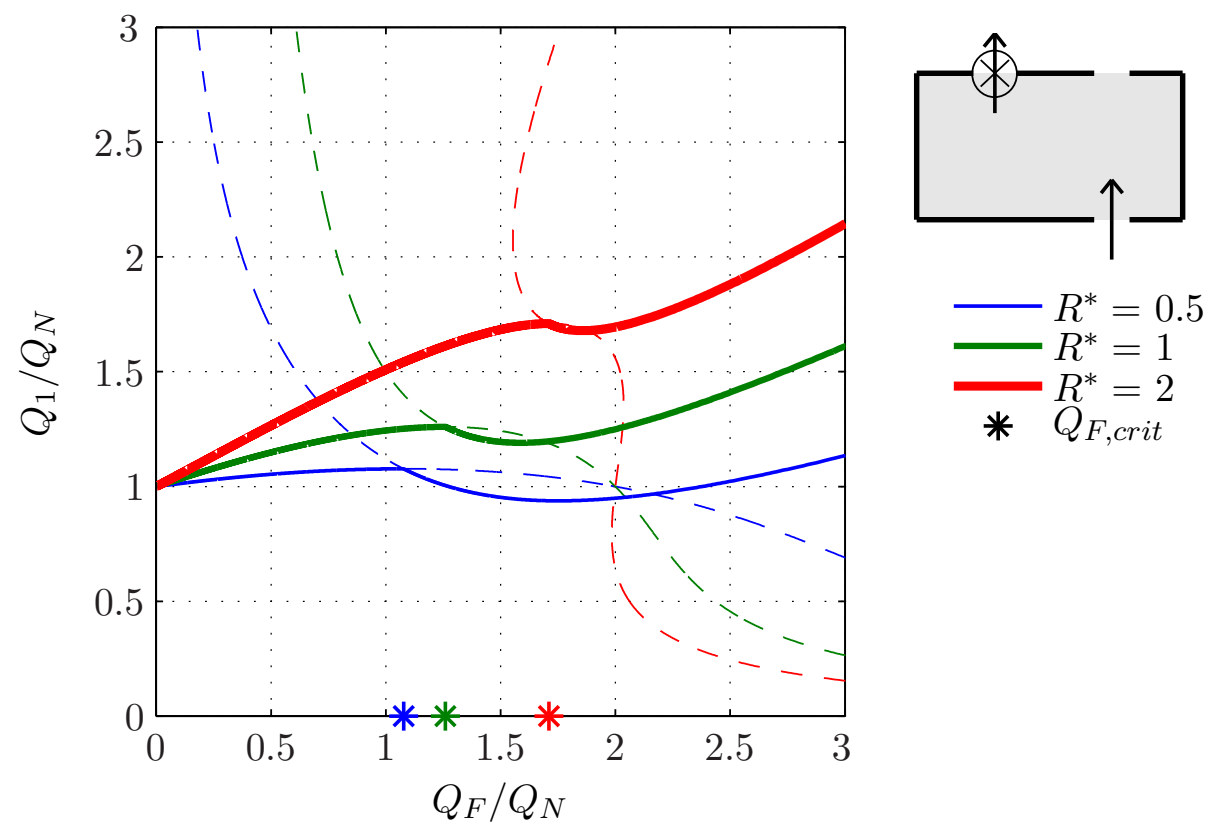

Figure 8: Hybrid extract ventilation. The floor-level volume flux, $Q_{1} / Q_{N}$, plotted as a function of forced extract volume flux, $Q_{F} / Q_{N}$, for three values of the effective vent area ratio, $R^{*}$.

ventilation reduces the pressure difference across the ceiling-level vent by exactly the same magnitude.

Rather than plotting the variation of $Q_{t o t} / Q_{N}$, we illustrate the variation of $g^{\prime} / g_{N}^{\prime}$, which expresses the relative temperature within the room (compared with purely natural ventilation). It was shown previously that

$$
\frac{g^{\prime}}{g_{N}^{\prime}}=\frac{Q_{N}}{Q_{t o t}}
$$

In figure $10, g^{\prime} / g_{N}^{\prime}$ is plotted against $Q_{F} / Q_{N}$ for three values of $R^{*}$. In the forward flow region, the figure shows the solution of (45). In the reverse flow region, $Q_{F}>Q_{F, \text { crit }}$, we plot the solution of (51).

We identify that for $Q_{F}<Q_{F, \text { crit }}$ there is a dependence on $R^{*}$, which is implied through the dependence of $g^{\prime} / g_{N}^{\prime}$ on $Q_{1} / Q_{N}$ in (45). For $Q_{F}>Q_{F, \text { crit }}$, however, there is no dependence on $R^{*}$, which is consistent with the solution of (51). If $R^{*}=0.5$, increasing $Q_{F} / Q_{N}$ from 0 to 1 produces a small reduction in $g^{\prime} / g_{N}^{\prime}$. For $R^{*}=2$, however, the same increase in $Q_{F} / Q_{N}$ results in a much greater reduction in $g^{\prime} / g_{N}^{\prime}$

The $R^{*}=1$ line in figure 10 is identical to the $R^{*}=1$ line in figure 6 , which illustrated hybrid supply ventilation. For $R^{*} \neq 1$, however, the two figures differ. In hybrid supply ventilation, the most effective cooling (smaller $g^{\prime} / g_{N}^{\prime}$ ) was provided by small values of $R^{*}$. 


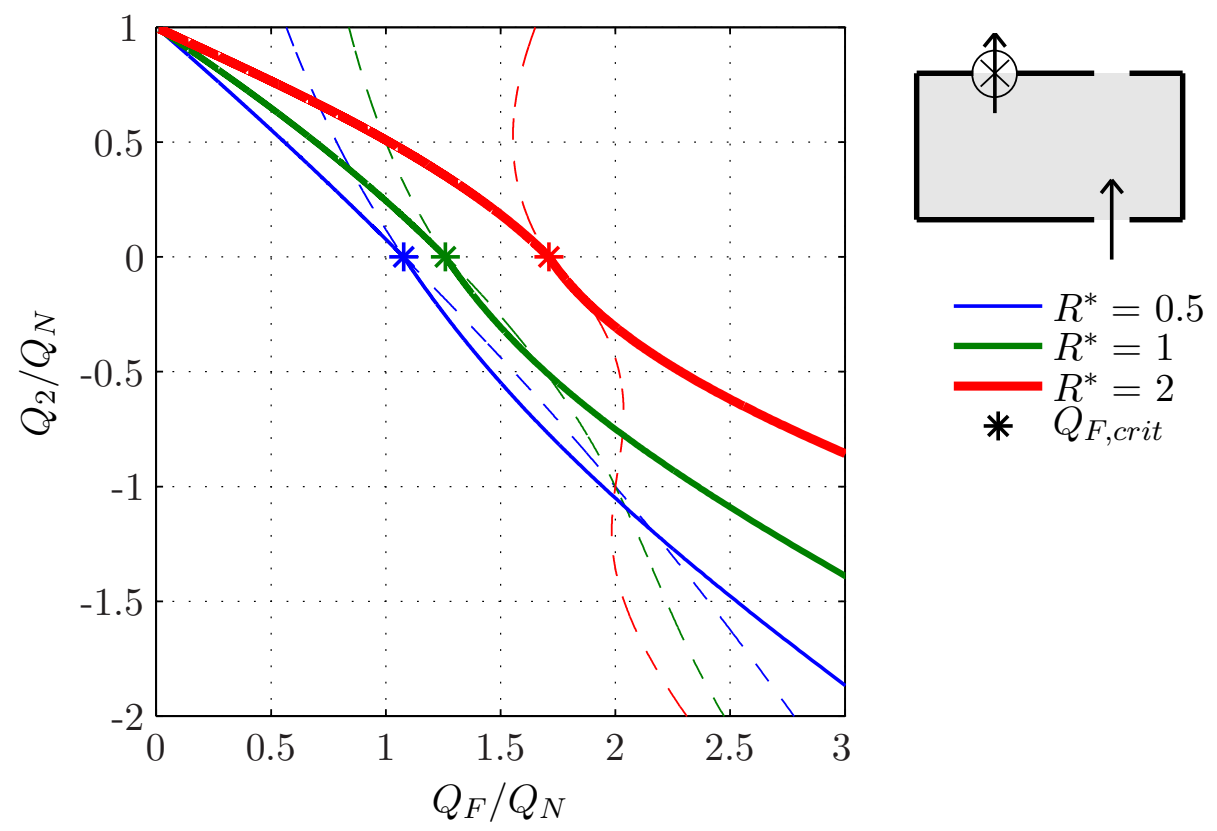

Figure 9: Hybrid extract ventilation. The ceiling-level volume flux, $Q_{2} / Q_{N}$, plotted as a function of forced extract volume flux, $Q_{F} / Q_{N}$, for three values of the effective vent area ratio, $R^{*}$.

In hybrid extract ventilation, larger values of $R^{*}$ result in more effective cooling; represented by a greater reduction in $g^{\prime} / g_{N}^{\prime}$ for a given increase in $Q_{F} / Q_{N}$.

In figure 11 , the position of the neutral pressure level, $z_{n p l} / H$, is plotted as a function of forced extract volume flux. For $Q_{F} / Q_{N}=0$, the purely natural ventilation solution, $z_{n p l} / H=1 /\left(1+R^{* 2}\right)$, is recovered. As $Q_{F} / Q_{N}$ is increased, the neutral pressure level moves higher in the room (closer to the ceiling). For $Q_{F}>Q_{F, \text { crit }}$, there is no physical neutral pressure level. The virtual neutral pressure level, $z_{n p l}$ (virtual), is represented by thin dashed lines in the region $z_{n p l} / H>1$.

For $R^{*}=0.5$, increasing the extract volume flux from $Q_{F} / Q_{N}=0$ to $Q_{F} / Q_{N}=1$ causes the neutral pressure level to move upwards from $z_{n p l} / H=0.8$ to $z_{n p l} / H=1$. For $R^{*}=2$, increasing $Q_{F} / Q_{N}$ from 0 to 1 results in an increase from $z_{n p l} / H=0.2$ to $z_{n p l} / H=0.7$, representing a much greater change. This comparison, between $R^{*}=$ 0.5 and $R^{*}=2$ in terms of the neutral pressure level, explains the different impact of a unit increase in $Q_{F} / Q_{N}$, which was described previously in terms of volume fluxes and local buoyancies.

\subsection{Hybrid extract ventilation - summary}

To summarise this section on hybrid extract ventilation: 


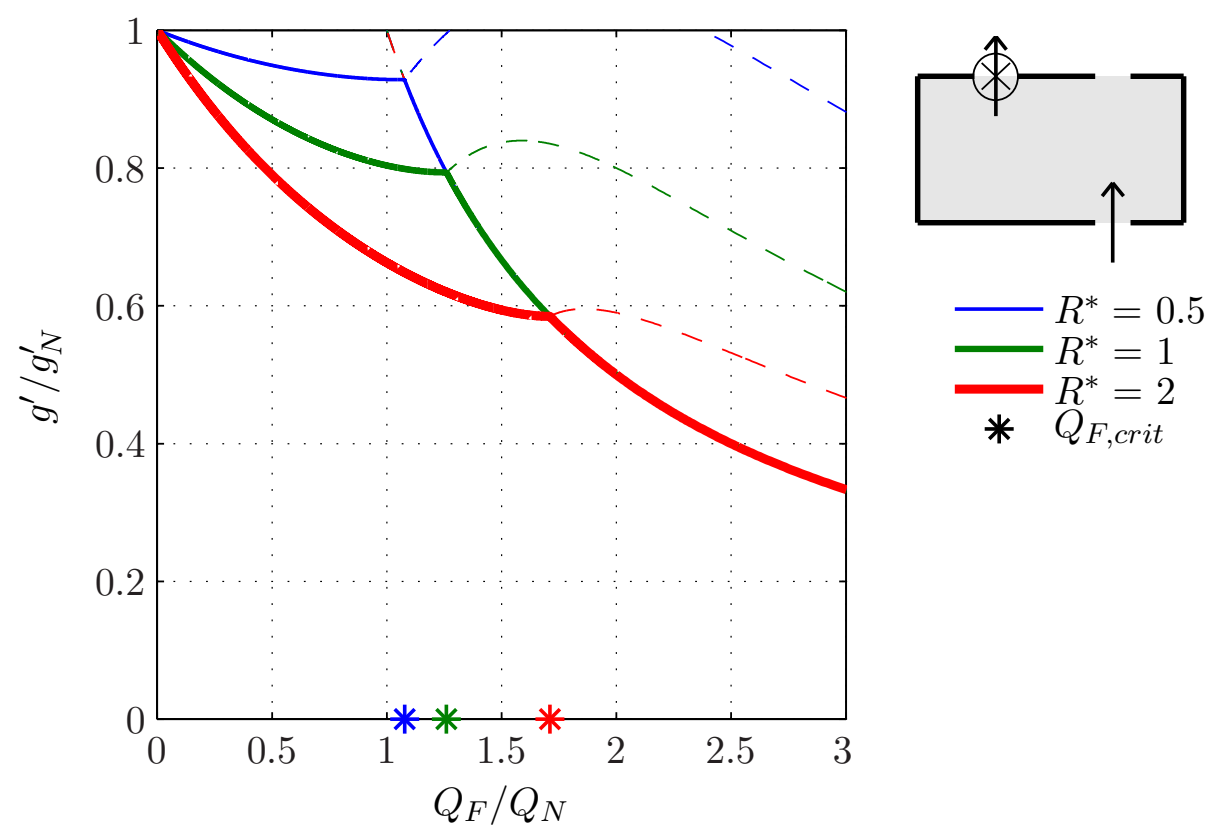

Figure 10: Hybrid extract ventilation. The uniform local buoyancy, $g^{\prime} / g_{N}^{\prime}$, plotted as a function of forced extract volume flux, $Q_{F} / Q_{N}$, for three values of the effective vent area ratio, $R^{*}$.

- the effect of a forced extract volume flux is to decrease the air pressure within a ventilated room;

- this results in the neutral pressure level moving upwards, i.e. towards the ceiling, when compared to its position in purely natural ventilation.

The effect of increasing $Q_{F} / Q_{N}$ in hybrid extract ventilation is:

- $Q_{1} / Q_{N}$ generally increases, eventually tending to a constant gradient which depends on $R^{*}$;

- $Q_{2} / Q_{N}$ decreases monotonically, also tending to a constant gradient;

- $g^{\prime} / g_{N}^{\prime}$ decreases, and for $Q_{F}>Q_{F, \text { crit }}$ the solution is independent of $R^{*}$;

- these effects are greater if $R^{*}$ is large.

We note that the behaviours summarised above are in stark contrast to those for hybrid supply ventilation (§3.5). 


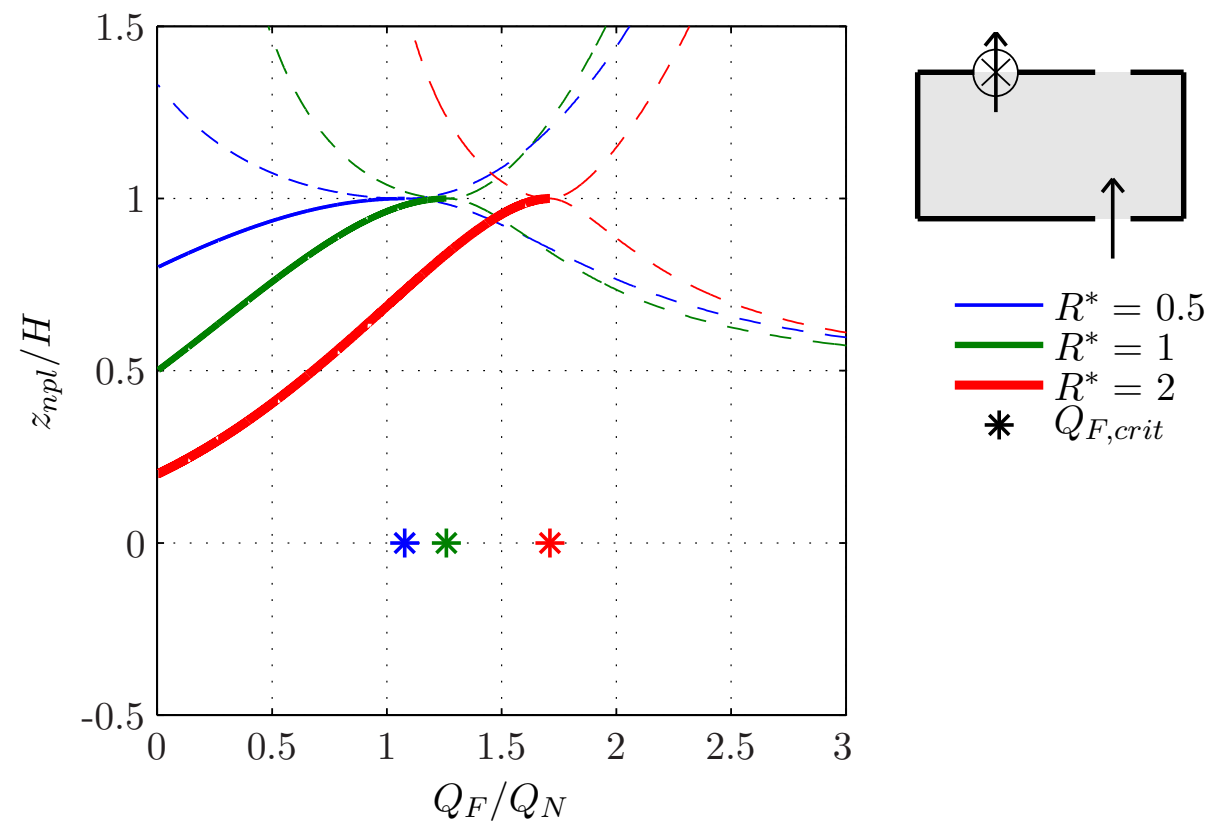

Figure 11: Hybrid extract ventilation. The position of the neutral pressure level, $z_{n p l} / H$, plotted as a function of forced extract volume flux, $Q_{F} / Q_{N}$, for three values of the effective vent area ratio, $R^{*}$. Dashed lines in the region $z_{n p l} / H>1$ and $Q_{F}>Q_{F . c r i t}$ are the reverse flow solution, (52), and give an indication of the change required in $Q_{F} / Q_{N}$ to return to forward flow.

\section{Implications for the design of hybrid ven- tilation strategies}

We have considered the idealised case of a uniform temperature, ventilated room with no heat transfer to or from the building fabric.

Regarding the choice of supply fan or extract fan, we find that for $R^{*}<1$, a supply fan provides greater enhancement of the ventilation, whilst for $R^{*}>1$, an extract fan provides better ventilation, this for a given fan volume flux, $Q_{F}$.

Regarding the likelihood of flow reversal: because a supply fan moves the neutral pressure level towards the floor, $z_{n p l} / H \downarrow$, flow reversal is more likely with large floor-level vents, i.e. $R^{*}>1$. Conversely, because an extract fan moves the neutral pressure level towards the ceiling, $z_{n p l} / H \uparrow$, flow reversal is more likely with large ceiling-level vents, i.e. $R^{*}<1$. 


\subsection{An example calculation}

Thus far, predictions have been presented in a general, non-dimensional form. In order to demonstrate the applicability of these predictions, we now present a worked, dimensional example.

Let us consider an isolated room, in which the floor-level vents are twice the area of the ceiling-level vents, so that $R^{*}=2$. Given the implications described above, we recommend the use of an extract fan rather than a supply fan, as this provides greater enhancement of the ventilation for large $R^{*}$ and delays the onset of reverse flow.

We consider a typical small office, with room height $H=3 \mathrm{~m}$, floor area $S=40 \mathrm{~m}^{2}$ and effective vent area $A^{*}=0.15 \mathrm{~m}^{2}$. Suppose the room receives $1 \mathrm{~kW}$ total heat input and the outside air temperature is taken to be $20^{\circ} \mathrm{C}$. First we calculate the natural ventilation volume flux,

$$
Q_{N}=\left(A^{* 2} B H\right)^{1 / 3}=0.12 \mathrm{~m}^{3} \mathrm{~s}^{-1} \equiv 3.7 \mathrm{ACH},
$$

where ACH denotes air changes per hour. The corresponding uniform local buoyancy is

$$
g_{N}^{\prime}=\left(\frac{B^{2}}{A^{* 2} H}\right)^{1 / 3}=0.23 \mathrm{~ms}^{-2}
$$

Thus, substituting values into (2), the room temperature in purely natural ventilation will be $26.7^{\circ} \mathrm{C}$.

We can now calculate the reduction in the temperature of interior air achieved by the addition of an extract fan. If we set the extract fan volume flux to be equal to the natural ventilation volume flux, $Q_{F}=3.7 \mathrm{ACH}$, then $Q_{F} / Q_{N}=1$ and, reading from the $R^{*}=2$ line in figure 10 , we find that,

$$
\frac{g^{\prime}}{g_{N}^{\prime}}=0.66
$$

indicating that the room temperature will be significantly cooler in hybrid extract ventilation, when compared with purely natural ventilation. Substituting this result into (23), the total hybrid ventilation volume flux can be calculated:

$$
Q_{t o t}=0.18 \mathrm{~m}^{3} \mathrm{~s}^{-1} \equiv 5.6 \mathrm{ACH} .
$$

Again substituting values into (2), the room temperature in steady hybrid extract ventilation can be calculated as $24.5^{\circ} \mathrm{C}$, representing a $2.2^{\circ} \mathrm{C}$ temperature reduction when compared to purely natural ventilation.

It is interesting to note that if the same fan were employed to supply $3.7 \mathrm{ACH}$ (rather than extract) it would achieve less than $0.5^{\circ} \mathrm{C}$ temperature reduction. This result emphasises the crucial importance of understanding the difference between hybrid supply and hybrid extract ventilation. 


\section{Conclusions}

This study has demonstrated that a supply fan and an extract fan at identical settings in identical rooms, do not necessarily have the same effect on the ventilation. This was established by theoretical investigation of hybrid ventilation of a room, in which buoyancy-induced and mechanically-imposed pressure differences combine to determine the resulting ventilation airflow. The exposition herein has demonstrated how the physical problem can be reduced to a mathematical model with only two key parameters; namely, the ratio of effective vent areas, $R^{*}$, and the ratio of mechanical to natural volume fluxes, $Q_{F} / Q_{N}$.

Through analysing solutions of our model, it was identified that, for $R^{*}>1$ (i.e. a room in which the floor-level vents are larger than the ceiling-level vents), an extract fan provides the most effective ventilation enhancement. This is because large values of $R^{*}$ cause the neutral pressure level to be generally low down in the room (close to the floor), and the effect of an extract fan is to raise the neutral pressure level away from the floor. In contrast, for small values of $R^{*}$, the neutral pressure level is generally high up in the room (close to the ceiling). Thus, if $R^{*}<1$, it is sensible to employ a supply fan, as this tends to lower the neutral pressure level, and provides the most effective enhancement of the ventilation in this situation.

\section{References}

Batchelor, G. K. 1954 Heat convection and buoyancy effects in fluids. Quarterly Journal of the Royal Meteorological Society 80 (345), 339358.

Connick, O. 2013 The fluid mechanics of hybrid ventilation. PhD Thesis, Imperial College London.

Delsante, A. \& Aggerholm, S. 2002 The use of simulation tools to evaluate hybrid ventilation control strategies, IEA Annex 35 Technical Report.

El Mankibi, M., Cron, F., Michel, P. \& Inard, C. 2006 Prediction of hybrid ventilation performance using two simulation tools. Solar Energy 80(8), 908-926.

Etheridge, D.W. \& Sandberg, M. 1996 Building Ventilation: Theory and Measurement. John Wiley \& Sons, Chichester.

Gao, R., Li, A., Hao, X., Lei, W., Zhao, Y. \& Deng, B. 2012 Fireinduced smoke control via hybrid ventilation in a huge transit terminal subway station. Energy and Buildings 45, 280-289.

Gladstone, C. \& Woods, A. W. 2001 On buoyancy-driven natural ventilation of a room with a heated floor. Journal of Fluid Mechanics 441, 293314.

Heiselberg, P. 2002 Principles of Hybrid Ventilation. Aalborg, Denmark: Aalborg University.

Hunt, G. R. \& Holford, J. M. 2000 The discharge coefficient experimental measurement of a dependence on density contrast. In 21st AIVC Annual Conference, Innovations in Ventilation Technology. The Hague, Netherlands. 
Hussain, S. \& Oostuizen, P.H. 2012 Validation of numerical modelling in an atrium space with a hybrid ventilation system. Building and Environment 52, 152-161.

IEA (1997) Annex 35 HybVent, HYBVENT- hybrid ventilation in new and retrofitted office buildings.

Jeong, Y. \& Haghighat, F. 2002 Modelling of a hybrid-ventilated building using ESPr. International Journal of Ventilation 1(2), 127139.

Ji, Y., Lomas, K. J. \& Cook, M. J. 2009 Hybrid ventilation for low energy building design in South China. Building and Environment 44 (11), 22452255 .

Kwon, O-H., Kim, M-H., Choi A-S. \& Jeong J-W. 2013 Energy saving potential of a hybrid ventilation system integrated with heat storage material. Energy and Buildings 57, 346-353.

Li, Y., Haghighat, F., Andersen, K.T., Brohus, H., Heiselberg, P.K., Dascalaki, E., Fracastoro, G.V., \& Perino, M. 1999 Analysis methods for natural and hybrid ventilation: an IEA ECB Annex 35 Literature Review. Aalborg: Department of Mechanical Engineering, Aalborg University.

Li, Y. 2002 Analysis of natural ventilation - a summary of existing analytical solutions. Tech. Rep. International Energy Agency, Annex 35, Hybvent.

Li, Y. \& Heiselberg, P. 2003 Analysis methods for natural and hybrid ventilation a critical literature review and recent developments. International Journal of Ventilation 1(4), 3-20.

Linden, P. F. 1999 The fluid mechanics of natural ventilation. Annual Review of Fluid Mechanics 31 (1), 201238.

Lomas, K.J., Cook, M.J. \& Fiala, D. 2007 Low energy architecture for a severe US climate: design and evaluation of a hybrid ventilation strategy. Energy and Buildings 39(1), 32-44.

Niachou, K., Hassid, S., Santamouris, M. \& Livada, I. 2008 Experimental performance investigation of natural, mechanical and hybrid ventilation in urban environment. Building and Environment 43(8), 1373-1382.

Tanaka, F., Majima, S., Kato, M. \& Kawabata, N. 2015 Performance validation of a hybrid ventilation strategy comprising longitudinal and point ventilation by a fire experiment using a model-scale tunnel. Fire Safety Journal 71, 287-298.

Wang, H. \& Chen, Q. 2013 A semi-empirical model for studying the impact of thermal mass and cost-return analysis on mixed-mode ventilation office buildings. Energy and Buildings 67, 267-274.

Wang, X, Jin, S., Ye, L.F. \& Lu, P. 2014 A study on the performance of natural wind in hybrid system in an atrium building. Applied Mechanics and Materials volumes 448-453, 1248-1255.

Zhai, Z., Johnson, M-H. \& Krarti, M. 2011 Assessment of natural and hybrid ventilation models in whole-building energy simulations. Energy and Buildings 43 (9), 22512261. 